\title{
I Need Help, not Likes: Review of Models to Detect Depression through Social Media Data
}

\author{
Ajay Agarwal \\ Dept. of Computer Science \& Engineering \\ DIT University, Dehradun \\ verslinfiniaudela@gmail.com
}

\begin{abstract}
Mental health is one of the most pressing challenges that we as humans are facing today. Despite the presence of various resources to detect and diagnose mental disorders, it continues to be an issue for a significant proportion of the world. Researchers are now exploring the application of natural language processing and deep learning on the diagnosis of these disorders.
\end{abstract}

However, due to the lack of a systematic and organized set of data to train upon, many of them have often failed. In this review paper, we explore prominent techniques to diagnose a specific mental disorder - Depression. We analyze and review the work done in the field of depression detection from feeds of social media platforms. For our study, we investigated mainly 3 platforms - Facebook, Twitter, and Reddit.

In the later sections of the paper, we aim to analyze another pressing topic of our study- The suitability of Social Media sources to act as data resources for the diagnostic tasks for mental health disorders. As the inclination of society towards social media increases, it evolves to become a better and robust outlet for people's emotions and expressions. Whilst recording and obtaining logs of a particular patient's visit with the therapist might be a more accurate description, however, it is one that is usually untimely and unlikely to be formally organized. In such scenarios, a patient's posts on various social media platforms and his activity on the same provide a better turn of perspective to solve such problems.

Keywords - RNN, CNN, Hierarchical Attention Networks, MILNET-architecture, Depression, Mental Health, embedding optimization

\section{Introduction}

According to the WHO (World Health Organization), mental health is: "a state of well-being in which the individual realizes his or her abilities, can cope with the normal stresses of life, can work productively and fruitfully, and can make a contribution to his or her community." The ability of a person to cope with the normal stresses of life is hampered when he or she is suffering from a mental disorder. Reports show that around $76 \%$ to $85 \%$ of people with mental disorders in low- and middle-income countries receive no treatment for their disorder. Often, the late diagnosis of mental disorder, lack of awareness regarding the importance of mental health, and the stigma regarding reaching out to therapists for seeking help/counseling is a large reason behind the increasing rates of stress, anxiety, and in even worst cases suicides among world's population.

Mental Disorders plaque almost every country in the world. No section of the population is not hit by some of the other mental disorders. Though, mental disorders comprise a broad range of problems, unlike the ubiquitous thinking, they come with various distinct symptoms which can be diagnosed distinctively. Most of these mental disorders can be treated. [2].

WHO (World Health Organization) defines mental disorders as “... (mental disorders) are generally characterized by some combination of abnormal thoughts, emotions, behavior, and relationships with others." It is to be noted that most of the mental disorders can be treated with diligent care and attention. In the next section, we shall talk about Depression providing a basic insight into the disorder. 


\section{Depression: The Silent Disorder}

One of the most significant contributors to the global burden of diseases is - Depression. It affects people in all the communities in the world. With over 350 million people as its victim, a recent World Mental Health Survey conducted in 17 countries discovered that about 1 in 20 people reports of suffering at least one episode of depression in the previous year. The most crucial thing to note is that depressive disorders don't have any specific age range of likelihood. Often, depression has been reported by pupils of young age, which over time results in a decline in their functioning, ability and ends them in becoming a liability than a resource. [10].

According to WHO, depression is defined as "a common mental disorder which is characterized by sadness, loss of interest or pleasure, feelings of guilt or low self-worth, disturbed sleep or appetite, feelings of tiredness and poor concentration". [8]. Depression has various severity stages; however, no stage can be considered to be less important. At its worst, depression can lead to suicide. [9].

\section{Mental Disorders and NLP}

Previous literature on mental disorder diagnose has successfully and oft acknowledged the increased complexity of the tasks and how the involvement of a manual agent is not a preference but a prerequisite.

With the rise of technology, social media has emerged to be a new attentive ear to the public's emotion. As a result, the conversational therapy has now been virtually outsourced to the internet for research to be conducted upon. To accurately diagnose a mental disorder, especially depression, one must realize that it stands crucial to first identify the key indicators in the content provided by the person. Even, if one has been successful in identifying the most prominent features and building a predictive model around it, increasing the accuracy of the model stands another major task. [12-13]

As a result of the above reasons, deep neural architectures are investigated for learning the features within the architecture itself.

\section{Research Methodology}

The theme for our investigation was a tripartite one. It is based on the review of the literature on depression detection through the feed which could be publicly obtained through social media platforms. We targeted the three most popular social media platforms - Twitter, Facebook, and Reddit.

To obtain the literature for our task, we decided to use keywords subjective to the platform understudy "depression detection twitter" + "depression detection facebook" + "depression detection Reddit". These keywords were queried in the Google Scholar platform [7] with the time range set as " $x$-to- $x$ ", where $x$ indicates the year under study. We studied the literature for the last three years- 2020, 2019, and 2018. As we investigated the literature, the most relevant works done in the field were listed in table 1.

Furthermore, we investigated the surge between awareness regarding depression detection among the public using the Google Trends platform.

\section{Observations}

Table 1 lists out the most relevant (cited) literature on depression detection based on the platform used as dataset arranged year wise.

\begin{tabular}{|c|c|c|c|}
\hline Platform & 2020 & 2019 & 2018 \\
\hline Twitter & $\begin{array}{l}\text { Vivek et al. } \\
\text {, Yan et al. } \\
\text {, Trifan et } \\
\text { al. , } \\
\text { Nurhaizatul } \\
\text { Jamil, Adil } \\
\text { Rajput }\end{array}$ & $\begin{array}{l}\text { Tong et } \\
\text { al., } \\
\text { Almouzini } \\
\text { et al., } \\
\text { Stephen et } \\
\text { al., Gui et } \\
\text { al., Leis et } \\
\text { al., Kumar } \\
\text { et al., } \\
\text { Bouarara, } \\
\text { Uddin et } \\
\text { al., } \\
\text { Burdisso } \\
\text { et al., } \\
\text { Farruque } \\
\text { et al., Tao } \\
\text { et al., }\end{array}$ & $\begin{array}{l}\text { Chen et al., } \\
\text { Zaghouani, } \\
\text { Biradar, Cong } \\
\text { et al., } \\
\text { Ravichandran, } \\
\text { Jacobson, } \\
\text { Kim et al., } \\
\text { Shrestha, } \\
\text { Sood et al., } \\
\text { Saxena, }\end{array}$ \\
\hline Facebook & $\begin{array}{l}\text { Marengo et } \\
\text { al. }\end{array}$ & $\begin{array}{l}\text { Ophir et } \\
\text { al., } \\
\text { Yamini et } \\
\text { al., }\end{array}$ & Islam et al., \\
\hline $\begin{array}{l}\text { Reddit } \\
\text { Post }\end{array}$ & $\begin{array}{l}\text { Correia et } \\
\text { al., } \\
\text { Alexander } \\
\text { \& Ruchi }\end{array}$ & $\begin{array}{l}\text { Tadesse et } \\
\text { al., Rijen } \\
\text { et al., Ji et } \\
\text { al., }\end{array}$ & $\begin{array}{l}\text { Stankevich et } \\
\text { al., Benamara } \\
\text { et al., Song et }\end{array}$ \\
\hline
\end{tabular}


Bataineh et al.,

al., Wang et al.,

Table 1. List of prominent works in the field of depression detection based on the platform used for the study

Figure 1 indicates the trend of depression detection using Internet search volume. This analysis was performed using the Google Trends platform for the keywords associated with depression detection. Also, the data for the present year (2020) has been excluded to keep the analysis more elaborate and comprehensive. ${ }^{*}$

"It is to be noted that data prior the year 2016 has not been taken as Google had made improvements to their data collection systems

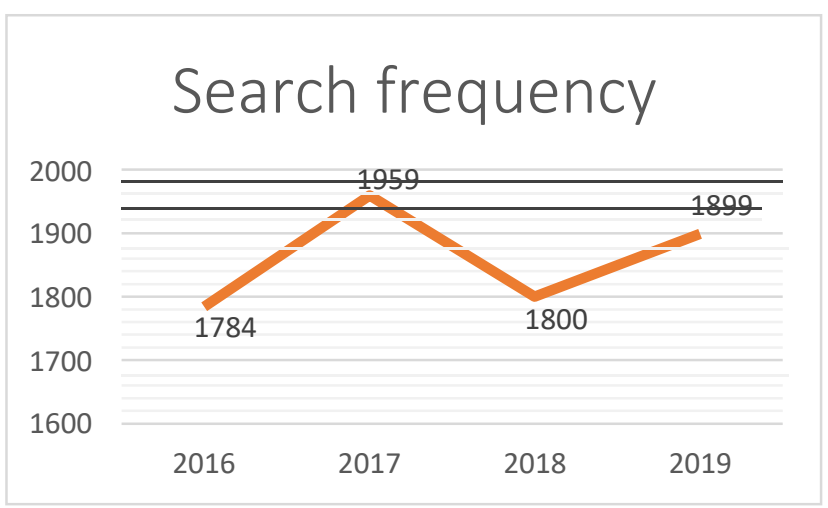

Figure 1. Search Frequency Trend on Google Search Platform regarding depression detection.

\section{Need Help, Not Retweets: Twitter Case-Study}

In this section, we review the methods utilized in the literature of the previous three years using Twitter as their dataset for the purpose.

Jacobson et al. [3] use the Naiyes Bayes classifier, Logistic Regression Classifier, SVM, and Random Forests for predicting whether the tweet provided by the user characterizes the user to be depressed or not. Around 3200 tweets were scraped for each verified user, mixed with an equal number of tweets from random Twitter users. F1 scores were recorded for a 10 -fold cross-validation metric used.
However, all these tweets were in the Korean language. The experiment showed that the Logistic Regression model performed better than the others assumed.

Around the same time, Ravichandran [4] developed the iMood. It was an automated depression detection tool based on the concept of supervised classification based sentiment analysis. The language of the dataset was English only. For the task of sentiment analysis, IBM Watson Tone Analyser was used. The results of iMood are unclear in the paper.

There exists a tremendous amount of literature on using Twitter as a dataset. However, it is often seen that the models used for the classification of tweets fail to follow the PHQ-9 standards for detection depression. While the text of the tweets is pre-processed, the context of the tweets isn't. As a result, the risk of having false-positive increases. [15].

To compare the models concisely, Figure 2 provides the accuracy of the classifiers used in the literature with a common or same evaluation metric.

\begin{tabular}{|l|l|r|}
\hline Work & Method Used & Accuracy \\
\hline Tong et al. & $\begin{array}{l}\text { Inverse Boosting } \\
\text { Pruning Trees }\end{array}$ & 0.9154 \\
\hline $\begin{array}{l}\text { Almouzini } \\
\text { et al. }\end{array}$ & Liblinear & 0.875 \\
\hline $\begin{array}{l}\text { Burdisso } \\
\text { et al }\end{array}$ & $\begin{array}{l}\text { SS3 classification } \\
\text { algorithm }\end{array}$ & 0.61 \\
\hline $\begin{array}{l}\text { Gui et al. } \\
\text { CNN+RL }\end{array}$ & 0.871 \\
\hline $\begin{array}{l}\text { Kumar et } \\
\text { al. }\end{array}$ & $\begin{array}{l}\text { Ensemble Vote } \\
\text { Classifier }\end{array}$ & 0.78 \\
\hline Saxena & PHQ-9 based classifier \\
\hline
\end{tabular}

Table 2. The above table depicts the name of the author, and the proposed framework used on the Twitter Dataset.

Whilst each author had presented a unique evaluation metric, accuracy was the one common all. 


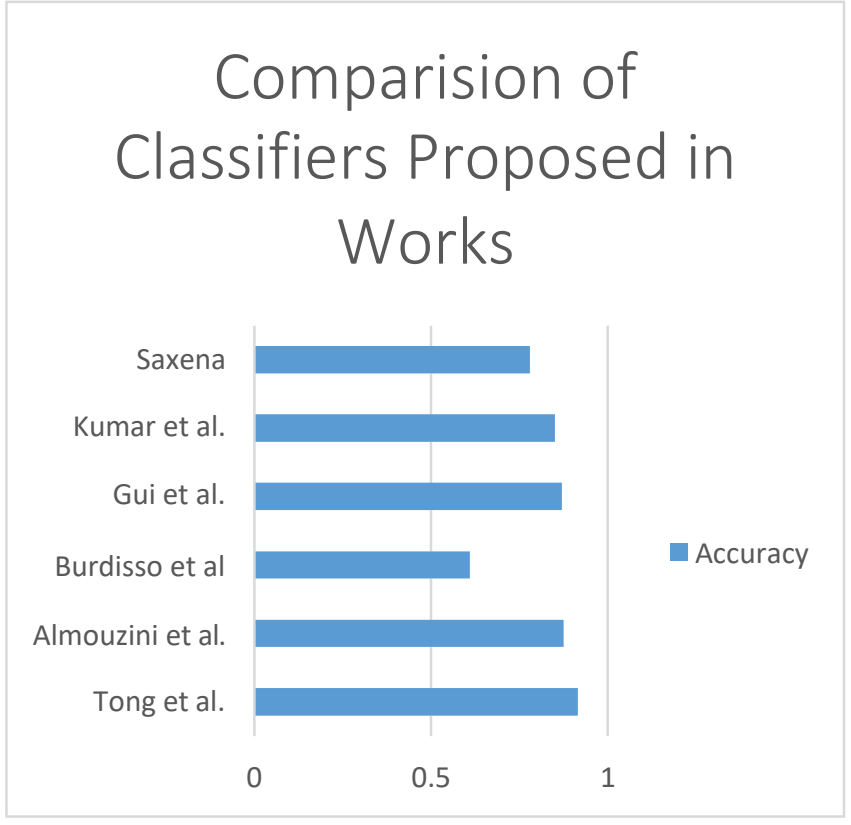

Figure 2. Graphical representation of the evaluation metric of the literature involved in the study

\section{Need Help, Not Reactions: Facebook Case Study}

In this section, we review the literature involving the use of Facebook posts and feed for the detection and classification of depression among the users.

Unlike the readily available Twitter API, Facebook has restricted access to individual user posts. The maximum access to public posts in such cases is based on the number of connections the investigator has from his/her own personal Facebook account. Due to the above reason, it can be fairly obvious that the literature using Facebook as a dataset is sparse as compared to Twitter.

In 2018, Islam et al. [5] investigated the use of KNN, Support Vector Machines, Decision Trees, and Ensemble Learning for depression detection. They made use of Facebook posts as their dataset which was obtained from an authentic online source.

Table 3 provides the list of the work done in the field involving Facebook posts.

\begin{tabular}{|l|l|}
\hline Work & Method Used \\
\hline Ophir et al. & $\begin{array}{l}\text { Content Analysis using } \\
\text { Beck depression } \\
\text { inventory-II }\end{array}$ \\
\hline
\end{tabular}

\begin{tabular}{|l|l|}
\hline Yamini et al & $\begin{array}{l}\text { Natural Language } \\
\text { Processing }\end{array}$ \\
\hline Islam et al & Decision Tree \\
\hline
\end{tabular}

Table 3. The above table lists out the models developed for detecting depression using Facebook as the dataset.

\section{Need Help, Not Upvotes: Reddit Case-Study}

In this section, we present the models that make use of the front page of the Internet- Reddit. Many datasets already exist based on Reddit posts. Some of them are also a part of the various ongoing challenges. To list a few- CELF eRisk and CLPsych.

In 2019, Rijen et al. [6] proposed the ensemble technique for the prediction of depression and its severity from the given Reddit posts. This model was the part of their solution to CLEF eRisk 2019. Their system leverages word polarities, extracted tokens using mutual information, expanded the keywords, and found semantic similarities for the classification of the Reddit Feed.

For the leveraging word polarities, Rijen et al. made use of the MPQA (Multi-Perspective Subjectivity Lexicon), having more than 8000 cues annotated with negative, neutral, and positive polarity. To consider for adding the mutual information $\mathrm{M}(\mathrm{X}, \mathrm{Y})$ joint probability density $\mu$ (x, y) based on entropy estimates from k-nearest neighbor distances from the sample of random points. The task of expanding the keywords and finding the semantic similarities was performed using a pre-trained GloVe embedding model. [16].

The model performed better than the baseline models in the field, however, showed a smaller score when compared against others using the DCHR Evaluation Metric. [11]

Table 4 lists the work done in the field on depression detection involving the use of Reddit posts along with the dataset used. Figure 3 shows the performance on basis of the precision evaluation metric for works listed in Table 4.

\begin{tabular}{l|ll} 
WORK & METHOD USED & DATASET \\
\hline $\begin{array}{l}\text { BENAMARA } \\
\text { ET AL. }\end{array}$ & $\begin{array}{l}\text { Supervised Learning } \\
\text { with Random Forest }\end{array}$ & eRisk \\
& 2017 \\
\end{tabular}




\begin{tabular}{l|ll} 
SONG ET AL. & $\begin{array}{l}\text { Feature Attention } \\
\text { Network }\end{array}$ & RSDD \\
\begin{tabular}{l|l} 
STANKEVICH \\
ET AL.
\end{tabular} & Random Forest & $\begin{array}{l}\text { eRisk } \\
2017\end{array}$ \\
$\begin{array}{l}\text { WANG ET } \\
\text { AL. }\end{array}$ & CNN with tf-idf & eRisk \\
& & 2018
\end{tabular}

Table 4. The above table lists the prominent works done in the field of depression detection using Reddit Posts.

Here, RSDD stands for the Reddit Self-reported Depression Diagnosis Dataset.

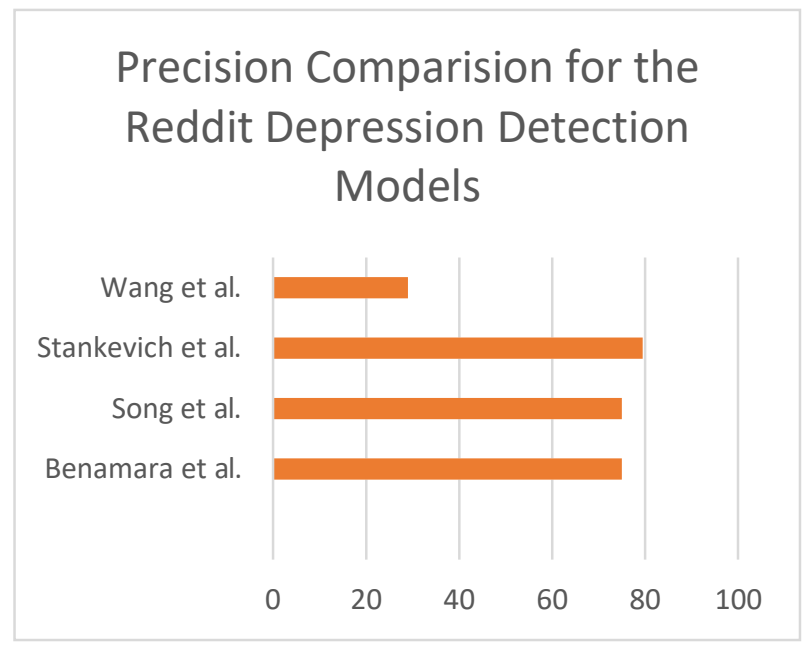

Figure 3. The above figure indicated the precision measure for the comparison of depression detection models making use of the social media platform- Reddit

\section{Future Work}

The future work for review involves including a more elaborate sense of reviewing. We can increase the timeline under survey and understand more analytically regarding how these models have evolved and helped us improve our understanding of depression from a computational perspective. [11].

The timeline can be increased and more models can be studied. We can also incorporate IoT based models under the review. Analogies can be drawn from the biological perspective of things to computational one.

\section{Conclusion}

The review of the literature on depression detection is a very exhaustive task. But, in the long run, shall prove to be a task of important sorts. This is because, only by such reviews, we can build upon better, more robust, and more accurate models to picture the human psyche. It is only then we can fully understand the brain - its nuances and its idiosyncrasies.

\section{References}

1. Google Trends. https://trends.google.com/trends/

2. Orabi, A. H., Buddhitha, P., Orabi, M. H., \& Inkpen, D. (2018, June). Deep learning for depression detection of twitter users. In Proceedings of the Fifth Workshop on Computational Linguistics and Clinical Psychology: From Keyboard to Clinic (pp. 8897).

3. 신효필, 남승호, \& 김문형. (2018). Detecting Language from Depressed Users with Korean Twitter Data (Doctoral dissertation, 서울대학교 대학원).

4. Ravichandran, S. (2018). iMood: Automated Depression Detection Tool.

5. Islam, M. R., Kabir, M. A., Ahmed, A., Kamal, A. R. M., Wang, H., \& Ulhaq, A. (2018). Depression detection from social network data using machine learning techniques. Health information science and systems, $6(1), 8$.

6. van Rijen, P., Teodoro, D., Naderi, N., Mottin, L., Knafou, J., Jeffryes, M., \& Ruch, P. (2019). A Data-Driven Approach for Measuring the Severity of the Signs of Depression using Reddit Posts.

7. Google Scholar. https://scholar.google.com/

8. World Health Organization. Depression and Other Common Mental Disorders: Global Health Estimates. http://apps.who.int/iris/bitstream/10665/25461 0/1/WHO-MSD-MER-2017.2-eng.pdf.

Published 2017. Accessed September 27, 2017. 
9. Marcus, Yasamy, Ommeren, Chisholm, \& Saxena. Depression - A Global Public Health Concern. WHO Department of Mental Health and Substance Abuse. https://www.who.int/mental_health/managem ent/depression/who_paper_depression_wfmh_ 2012.pdf. Published 2012.

10. World Health Organization. Depression. https://www.who.int/news-room/factsheets/detail/depression. Published 2017.

11. Kosinski, M., Matz, S. C., Gosling, S. D., Popov, V., \& Stillwell, D. (2015). Facebook as a research tool for the social sciences: Opportunities, challenges, ethical considerations, and practical guidelines. American Psychologist, 70(6), 543.

12. Maron, O., \& Lozano-Pérez, T. (1998). A framework for multiple-instance learning. In Advances in neural information processing systems (pp. 570-576).

13. Andrews, S., Tsochantaridis, I., \& Hofmann, T. (2003). Support vector machines for multipleinstance learning. In Advances in neural information processing systems (pp. 577-584).

14. Wang, Y., Long, M., Wang, J., Gao, Z., \& Philip, S. Y. (2017). Predrnn: Recurrent neural networks for predictive learning using spatiotemporal lstms. In Advances in Neural Information Processing Systems (pp. 879-888).

15. Hu, B., Lu, Z., Li, H., \& Chen, Q. (2014).

Convolutional neural network architectures for matching natural language sentences. In Advances in neural information processing systems (pp. 2042-2050).

16. Yin, W., Kann, K., Yu, M., \& Schütze, H. (2017). Comparative study of CNN and RNN for natural language processing. arXiv preprint arXiv:1702.01923. 